\title{
Surgical Relief of Lagophthamos following Seventh Nerve Paralysis in Cases of Leprosy.
}

H. H. Gass.

NYONE working in a leprosy settlement where there
are a considerable percentage of N3 cases, is well
aware of the distressing ocular conditions resulting from seventh nerve paralysis. The process does not always stop at the irritative stage but often goes on to corneal ulceration, irido-cyclitis, and finally to total destruction of the eye. In the more chronic cases, usually the first sign of corneal involvement is the vascularisation of the lower pole of the cornea. This is in contradistinction to trachoma, which usually shows pannus at the superior pole. Palliative treatment consisting of bandaging the affected eye, or eyes, at night with absorbent cotton soaked with liquid paraffin does give relief. But patients tire of this and get lax in continuing this form of treatment. A more permanent form of treatment is desired.

The operative procedure which we have found of value in Chandkhuri, is the one described in Oxford Looseleaf Surgery," Vol. 4, Part I., page 290, and is called Canthorrhaphy.

Indication: Paralysis of the orbicularis oculi which prevents the lids from completely covering the cornea.

Preparation: Iodine paint over skin surface of the lower lid which is to be excised. Thorough washing of the eye

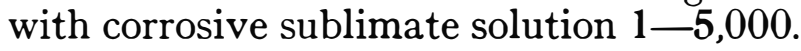

Anaesthesia: Two drops of $4 \%$ cocaine solution dropped into the eye every five minutes, three times. $1 \%$ novocaine solution for skin and subconjunctival infiltration. We find it useful to add a few drops of adrenaline, $1-1,000$ solution for the control of bleeding. We first evert the upper lid and inject the portion of conjunctiva which is to be excised 
with enough novocaine solution to produce moderate ballooning. The skin area of the lower lid is dealt with similarly.

Operation: Before beginning the operation it is advisable to determine where the new lateral canthus is to be. This point is marked on the upper and lower lids. The upper lid is now everted and an incision $\mathbf{5} \mathrm{mm}$. deep is made along the intermarginal line and carried out to the lateral canthus. Then an incision sufficiently deep to excise the conjunctiva is carried upwards for about five millimetres, after which it is carried out to the lateral canthus. The incision being in the form of a triangle with its base towards the medial part of the eye, and its apex at the lateral canthus. A similar incision is made over the skin of the lower lid as shown in diagram. It is very important at this time to remove the lash bearing area of the lower lid, which is to be included in the suture. I have had cases in which I failed to be thorough about this procedure with the result that lashes grew again with much discomfort to the patient. The union of the two corresponding raw surfaces is merely the matter of a mattress suture. The suture material which we use is silk. The suture should be armed with two fine eye needles with a full curve. The first needle with suture is passed from the under or conjunctival surface of the lower lid and out through the excised area of skin, then through the raw or under surface of the upper lid to the outside. This procedure is repeated with the needle at the other end of the suture at a distance of about $4 \mathrm{~mm}$. from the point of entry of the first needle. This procedure is illustrated in the following diagram :-

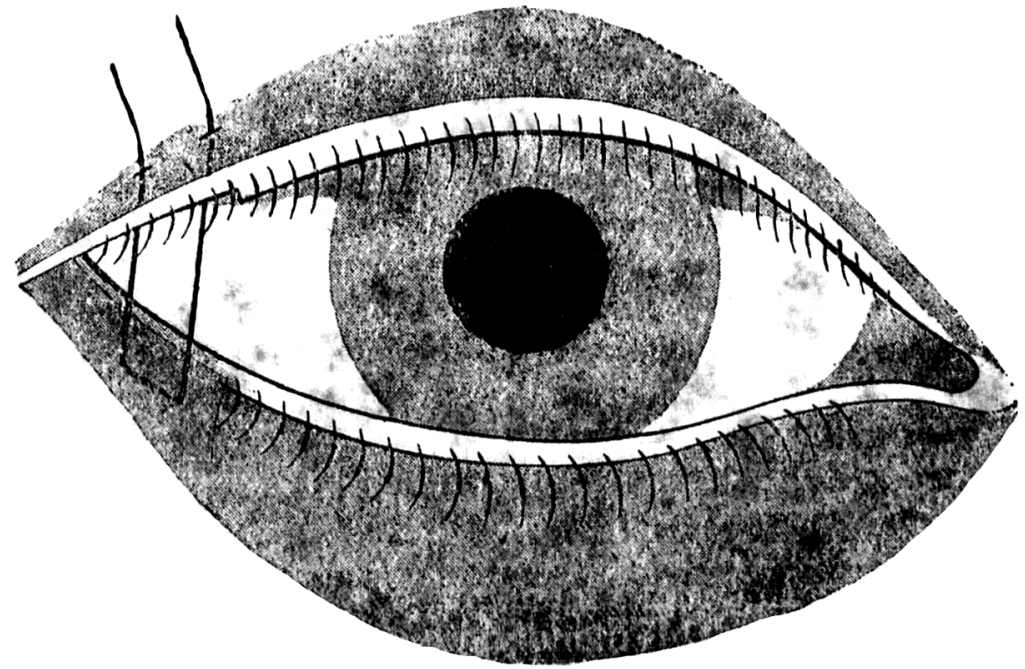

Reproduced by permission of Oxford University Press, New York 
It is wise to tie the two ends of the mattress stitch over a small ball of cotton so as to avoid cutting into the skin. A short continuous stitch may be taken to approximate the margins of the lids. This is, however, not necessary if there is proper approximation with the mattress stitch. The eye or eyes should be kept bandaged for about ten days. We usually remove the mattress stitch on the twelfth day. The eye may be washed with weak antiseptic solutions after the fourth or fifth day. Of course, the less meddling there is after the operation the less danger there is of non-union of the two surfaces. When dressings are changed, it is important to avoid any pulling or tugging. Before removing the eye-pad it should be thoroughly soaked with warm sterile boric lotion.

The cosmetic result of the operation is better if a bilateral canthorrhaphy is done. It is, however, not always necessary to do both sides. 